\title{
Distinct in vitro Complement Activation by Various Intravenous Iron Preparations
}

\author{
Julia Cordelia Hempel ${ }^{a}$ Felix Poppelaars $^{a}$ Mariana Gaya da Costa ${ }^{a}$ \\ Casper F.M. Franssen ${ }^{a}$ Thomas P.G. de Vlaam ${ }^{a}$ Mohamed R. Daha ${ }^{a}$ b \\ Stefan P. Berger ${ }^{\mathrm{a}}$ Marc A.J. Seelen ${ }^{\mathrm{a}}$ Carlo A.J.M. Gaillard ${ }^{\mathrm{a}}$ \\ ${ }^{a}$ Department of Internal Medicine, Division of Nephrology, University of Groningen, University Medical Center

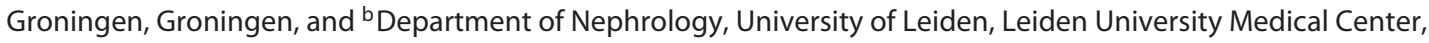 \\ Leiden, The Netherlands
}

\section{Key Words}

Intravenous iron - Complement activation related pseudo allergy · Hypersensitivity reaction · Complement activation · Iron sucrose $\cdot$ Iron dextran

\begin{abstract}
Background: Intravenous (IV) iron preparations are widely used in the treatment of anemia in patients undergoing hemodialysis (HD). All IV iron preparations carry a risk of causing hypersensitivity reactions. However, the pathophysiological mechanism is poorly understood. We hypothesize that a relevant number of these reactions are mediated by complement activation, resulting in a pseudo-anaphylactic clinical picture known as complement activation-related pseudo allergy (CARPA). Methods: First, the in-vitro complement-activating capacity was determined for 5 commonly used IV iron preparations using functional complement assays for the 3 pathways. Additionally, the preparations were tested in an ex-vivo model using the whole blood of healthy volunteers and HD patients. Lastly, in-vivo complement activation was tested for one preparation in HD patients. Results: In the in-vitro assays, iron dextran, and ferric carboxymaltose caused complement activation, which was only possible under alternative pathway conditions. Iron sucrose may interact with complement proteins, but did not activate complement in-vitro. In the ex-vivo assay, iron dex-
\end{abstract}

\section{KARGER}

E-Mail karger@karger.com www.karger.com/ajn

\section{The Author(s) \\ Published by S. Karger AG, Basel \\ Karger \\ Open access}

This article is licensed under the Creative Commons Attribution NonCommercial-NoDerivatives 4.0 International License (CC BYNC-ND) (http://www.karger.com/Services/OpenAccessLicense). Usage and distribution for commercial purposes as well as any distribution of modified material requires written permission. tran significantly induced complement activation in the blood of healthy volunteers and HD patients. Furthermore, in the ex-vivo assay, ferric carboxymaltose and iron sucrose only caused significant complement activation in the blood of HD patients. No in-vitro or ex-vivo complement activation was found for ferumoxytol and iron isomaltoside. IV iron therapy with ferric carboxymaltose in HD patients did not lead to significant in-vivo complement activation. Conclusion: This study provides evidence that iron dextran and ferric carboxymaltose have complement-activating capacities in-vitro, and hypersensitivity reactions to these drugs could be CARPA-mediated.

(C) 2016 The Author(s)

Published by S. Karger AG, Basel

\section{Introduction}

A majority of patients with chronic kidney disease (CKD) receive intravenous (IV) iron for the treatment of anemia [1]. However, controversy exists regarding the safety of IV iron preparations since hypersensitivity reactions have been reported for all iron drugs [2]. Although these reactions appear sporadic, they can be acute and life

C.J.H. and F.P. contributed equally.

Marc A.J. Seelen, MD, PhD

University Medical Center Groningen

Department of Internal Medicine, Division of Nephrology, AA53

Postbus 196, NL-9700 AD Groningen (The Netherlands)

E-Mail m.seelen@umcg.nl 
threatening. The exact frequency of the hypersensitivity reactions is unknown. This is attributed to a lack of data, due to underreporting and differential reporting [3].

The underlying mechanism of reactions due to the hypersensitivity of IV iron remains unclear. However, elucidating the pathophysiology is critical to improve prediction, prevention and management of these adverse events. In contrast to the immunoglobulin E (IgE)-mediated anaphylaxis observed in older compounds of IV iron, hypersensitivity reaction due to new IV iron preparations are thought to result from complement activation-related pseudo allergy (CARPA) $[4,5]$. Nonetheless, this has not been tested systematically. CARPA is an adverse event seen after the administration of monoclonal antibodies, intravenously administered drugs and nanoparticle-containing drugs $[4,5]$. CARPA was postulated since all available preparations consist of iron-carbohydrate nanoparticles [6].

Activation of the complement system occurs via 3 pathways: the classical pathway $(\mathrm{CP})$, the lectin pathway (LP) and the alternative pathway (AP). The CP is activated by antibody-antigen complexes, the LP by carbohydrates and the AP by microbial surfaces. This results in the formation of the C3-and C5-convertases and the generation of anaphylatoxins. Subsequently, activation of the terminal pathway leads to the formation of the membrane attack complex (C5b-9) [7]. In CARPA, such a cascade is initiated predominantly by the generation of complement activation products, leading to the stimulation of mast cells and basophil granulocytes resulting in secretion products, which cause various responses in effector cells such as platelets, endothelial cells and smooth muscle cells. Clinically, these processes may give rise to bronchospasm, laryngeal edema, tachycardia, hypo- or hypertension and hypoxia [5].

The aim of this study was to determine the effect of 5 currently available IV iron preparations on the complement system. By evaluating different IV iron drugs in an in-vitro and ex-vivo model for complement activation, we intended to test for the probability of CARPA by IV iron drugs. Lastly, in-vivo complement activation was tested for one IV iron preparation in hemodialysis (HD) patients.

\section{Materials and Methods}

\subsection{Subjects}

We recruited 2 groups:

- Control subjects (5-10 per experiment, as indicated below).

- Patients on maintenance HD $(n=8)$. During one dialysis session, blood samples were taken at 0,120 and 240 min dur- ing dialysis. Patients received $100 \mathrm{mg} / 2 \mathrm{ml}$ ferric carboxymaltose $\left(\right.$ Ferinject ${ }^{\odot}$ ) IV over $1 \mathrm{~h}$ at $120 \mathrm{~min}$ into the dialysis session.

\subsection{Reagents}

Iron sucrose $\left(\right.$ Venofer $\left.^{\odot}\right)$ and ferric carboxymaltose $\left(\right.$ Ferinject ${ }^{\oplus}$ ) were purchased from Vifor Nederland, Breda, The Netherlands; ferumoxytol $\left(\right.$ Rienso $^{\odot}$ ) from Takeda Nederland, Hoofddorp, The Netherlands; low molecular weight iron dextran $\left(\mathrm{CosmoFer}^{\odot}\right)$ and iron isomaltoside 1000 (Monofer $^{\odot}$ ) from Cablon Medical, Leusden, The Netherlands.

For the whole blood experiments, lepirudin (Refludan $^{\odot}$, Hoechst, Frankfurt am Main, Germany) was used as anticoagulant.

\subsection{Normal Human Serum}

Blood was taken from 10 healthy volunteers and directly stored on ice. Samples were centrifuged, then pooled and stored at $-80^{\circ} \mathrm{C}$ until further analysis.

\subsection{Complement Pathway Activity in Human Serum}

Functional assays were used to allow quantification of complement activation via the CP, the LP and AP in human serum. These assays were previously described [8]. In brief, 96-well plates were coated overnight with human immunoglobulin $M$ for the CP, mannan for the LP or lipopolysaccharide (LPS) for the AP. Plates were washed 3 times after each step with PBS containing $0.05 \%$ Tween-20. Plates were blocked with $1 \%$ bovine serum albumin (BSA) in PBS for $1 \mathrm{~h}$ at $37^{\circ} \mathrm{C}$. Serum was diluted in gelatin veronal buffer (GVB) buffer adapted specifically for each pathway. For the CP and LP, serum was diluted in GVB with $\mathrm{Ca}^{2+}-\mathrm{Mg}^{2+}$. For the AP, serum was diluted in GVB with magnesium only. After $1 \mathrm{~h}$ at $37^{\circ} \mathrm{C}$, deposition the of properdin, C4, C3 or C5b-9 was detected using rabbit anti-human properdin (obtained from the laboratory of Nephrology, Leiden, The Netherlands), mouse anti-human C4 (obtained from the laboratory of Nephrology, Leiden, The Netherlands), RFK22 (anti-human C3, obtained from the laboratory of Nephrology, Leiden, The Netherlands) and AE11 (anti-human C5b-9, DAKO, Glostrup, Denmark), respectively. Binding of antibodies was detected using the appropriate primary and secondary antibody. For visualization, TMB and $\mathrm{H}_{2} \mathrm{SO}_{4}$ were added before the absorption was measured at $450 \mathrm{~nm}$.

Prior to incubation on the enzyme linked immunosorbent assay (ELISA) plate, all serum samples were pre-incubated at $37^{\circ} \mathrm{C}$ for $30 \mathrm{~min}$ with iron in a dose ranging from 0.0625 to $0.5 \mathrm{mg} / \mathrm{ml}$. Next, samples were further diluted to the final concentration with the appropriate buffer.

\subsection{Complement Activation Assays by IV Iron}

For the complement activation assay, iron preparations or BSA were coated overnight on a 96-well plate followed by blocking with $1 \% \mathrm{BSA} / \mathrm{PBS}$ at $37^{\circ} \mathrm{C}$ for $1 \mathrm{~h}$. The wells were exposed to pooled human serum diluted in adapted GVB (see 2.3) or with ethylenediaminetetraacetic acid (EDTA; $20 \mathrm{mM}$ ) for $1 \mathrm{~h}$ at $37^{\circ} \mathrm{C}$. The plate was then incubated with antibodies against properdin, C3 or C5b-9 (see 2.3). Detection was completed using appropriate primary and secondary antibody. The plate was washed with PBS Tween-20 (0.05\%) after each step. Visualization was similar as described in section 2.3 .
Hempel et al. 
Table 1. Activation of complement components of the CP, LP and AP by IV iron drugs

\begin{tabular}{|c|c|c|c|c|c|c|c|}
\hline \multirow{2}{*}{$\begin{array}{l}\text { Residual complement } \\
\text { activity }^{1}\end{array}$} & \multicolumn{7}{|c|}{ IV iron preparations, $\%$} \\
\hline & control & iron dextran & ferric carboxy-maltose & $\begin{array}{l}\text { iron isomaltoside } \\
1000\end{array}$ & ferumoxytol & iron sucrose & \\
\hline $\mathrm{CP}$ & $100 \pm 5.5$ & $126.8 \pm 23.4$ & $94.3 \pm 13.8$ & $108.2 \pm 3.9$ & $110.8 \pm 4.2$ & $10.4 \pm 4.5^{* * *}$ & \\
\hline LP & $100 \pm 5.2$ & $88 \pm 4.4$ & $88.7 \pm 3.5$ & $84.3 \pm 2.4$ & $91.4 \pm 3.3$ & $4.7 \pm 0.4^{* * *}$ & \\
\hline \multirow{2}{*}{$\begin{array}{l}\text { Complement } \\
\text { activation }^{2}\end{array}$} & \multicolumn{7}{|c|}{ IV iron preparations, $\%$} \\
\hline & $\begin{array}{l}\text { positive } \\
\text { control }\end{array}$ & iron dextran & ferric carboxy-maltose & $\begin{array}{l}\text { iron isomaltoside } \\
1000\end{array}$ & ferumoxytol & iron sucrose & BSA \\
\hline $\mathrm{CP}$ & $100 \pm 0.5$ & $2.9 \pm 0.1$ & $2.5 \pm 0.1$ & $2.5 \pm 0.1$ & $3.0 \pm 0.1$ & $3.0 \pm 0.0$ & $2.9 \pm 0.1$ \\
\hline
\end{tabular}

\subsection{Complement Pathway Activity in Human Whole Blood}

The experimental set-up has previously been described [9]. In short, blood was drawn in LPS-free tube with $50 \mu \mathrm{g} / \mathrm{ml}$ lepirudin. Whole blood was then incubated for 0 or $90 \mathrm{~min}$ at $37^{\circ} \mathrm{C}$ with IV iron $(0.5 \mathrm{mg} / \mathrm{ml}$ ferrous iron) while continuously rotated. PBS was added to the negative controls. The reaction was stopped with EDTA (final concentration of $20 \mathrm{~mm}$ ). Samples were then centrifuged and plasma was stored at $-80^{\circ} \mathrm{C}$ until further analysis.

2.7 Quantification of the Antigenic Levels of C1q, C3d, C3

Mannose-Binding Lectin, Properdin and C5b-9

The ELISA for C1q, C3d, C3 mannose-binding lectin (MBL), properdin, and C5b-9 were performed as described previously [10-12].

\subsection{Statistics}

Statistical analyzes were performed using BM SPSS Statistics Version 22 and $\mathrm{p}$ values $<0.05$ were considered statistically significant. The Kruskal-Wallis test and Mann-Whitney U test were used to assess differences between groups of non-parametric data and one-way analysis of variance and t test for normally distributed data. If needed, data was in-transformed for normality.

\subsection{Ethics}

All participants gave informed consent. The Medical Ethical Committee of the University Medical Center Groningen has reviewed the study design and it was confirmed that an official approval of this study by the committee is not required since the Medical Research Involving Human Subjects Act (WMO) does not apply.

Complement Activation by IV Iron

\section{Results}

\subsection{In-vitro effect of IV iron preparations on complement activity}

The interaction of the different IV iron drugs with complement was determined using functional complement assays for each pathway. Normal human serum (NHS) was pre-incubated with different IV iron drugs prior to the assay; subsequently, residual complement activity was measured. In this assay, decreased residual activity reflects either activation or inhibition of complement by the IV iron compound during the pre-incubation period.

\subsubsection{Decreased Residual Activity of the CP by Iron}

Sucrose

First, residual complement activity was tested for the CP after incubation with the IV iron drugs (table 1). Iron sucrose was the only preparation that significantly reduced residual complement activity. Furthermore, the effect of iron sucrose on CP activity $(\mathrm{p}=0.016)$ was dose-dependent (fig. 1). At a concentration of 0.5 $\mathrm{mg} / \mathrm{ml}$, iron sucrose reduced C4, C3 and C5b-9 deposition by 92,88 and $96 \%$, respectively ( $p<0.001)$. For iron dextran, ferric carboxymaltose, iron isomaltoside 


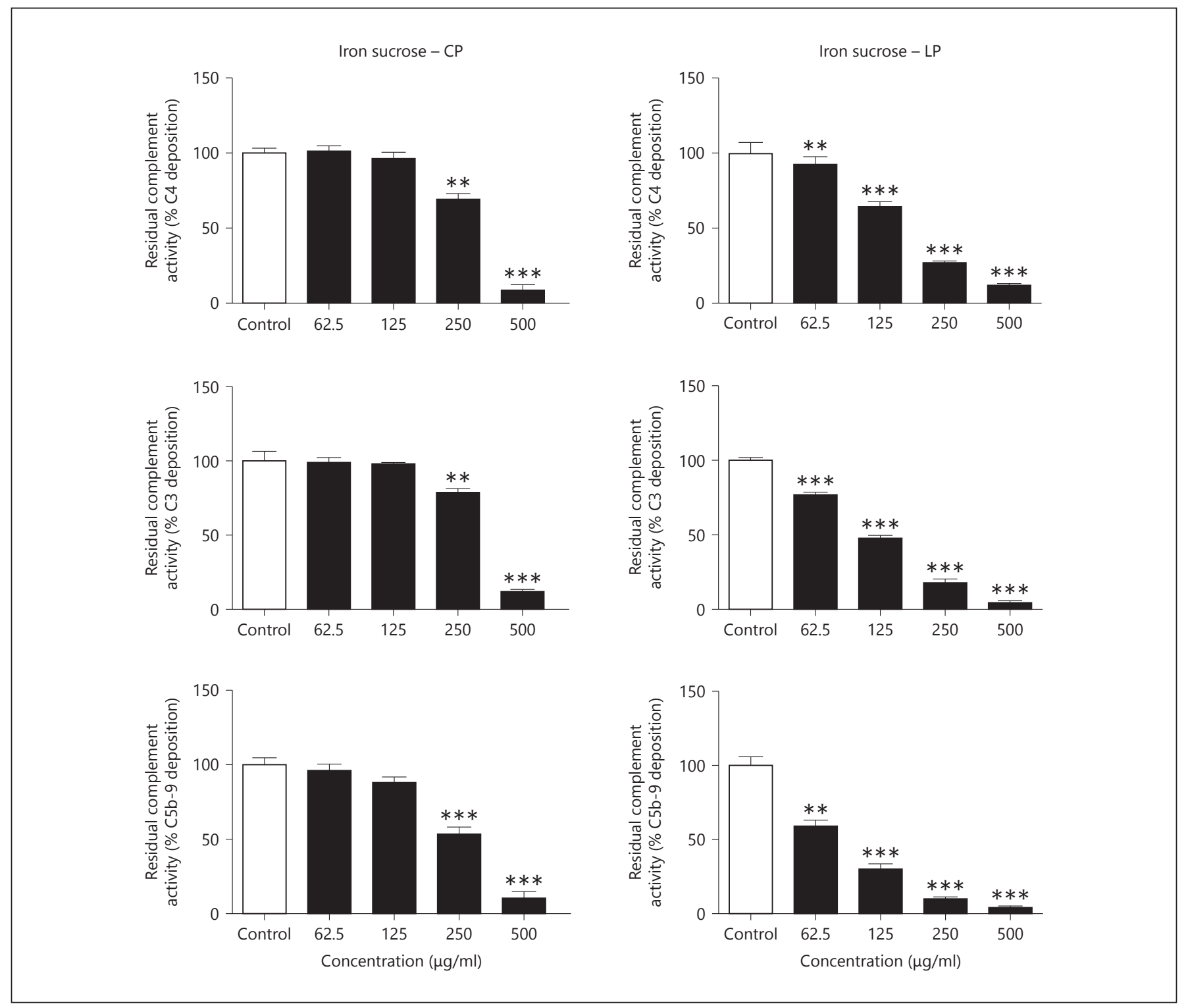

Fig. 1. The dose-dependent decrease of residual activity of the CP, LP and AP by iron sucrose, iron dextran and ferric carboxymaltose. Pooled serum was pre-incubated with increasing concentrations of intravenous iron (x-axis, $\log 2$ scale) for $30 \mathrm{~min}$ at $37^{\circ} \mathrm{C}$. PBS was used for the controls. The serum was then used in the functional assay for the CP, LP and AP to measure residual activity. Deposition of C4, properdin, C3 and C5b-9 were used as readout and the amount obtained in the control was set at $100 \%$ (yaxis). Data are shown as mean \pm SEM of 3 experiments $(* \mathrm{p}<0.05$, $\left.{ }^{* *} \mathrm{p}<0.01,{ }^{* * *} \mathrm{p}<0.001\right)$.

(Figure continued on next page.) and ferumoxytol, there was no change in residual complement activity, indicating low to no effect on the CP.

\subsubsection{Decreased residual activity of the LP by iron sucrose}

Next, residual complement activity for the LP was assessed (table 1). Once again, iron sucrose significantly re- duced residual complement activity in a dose-dependent manner $(\mathrm{p}<0.001)$ indicating prominent activation of the LP during the pre-incubation period (fig. 1). Deposition of C4, C3 and C5b-9 were lowered by 88,95 and $95 \%$ at $0.5 \mathrm{mg} / \mathrm{ml}$ for iron sucrose $(\mathrm{p}<0.001)$. For iron dextran, ferric carboxymaltose, iron isomaltoside and ferumoxytol, there was no change in residual complement activity for the LP. 


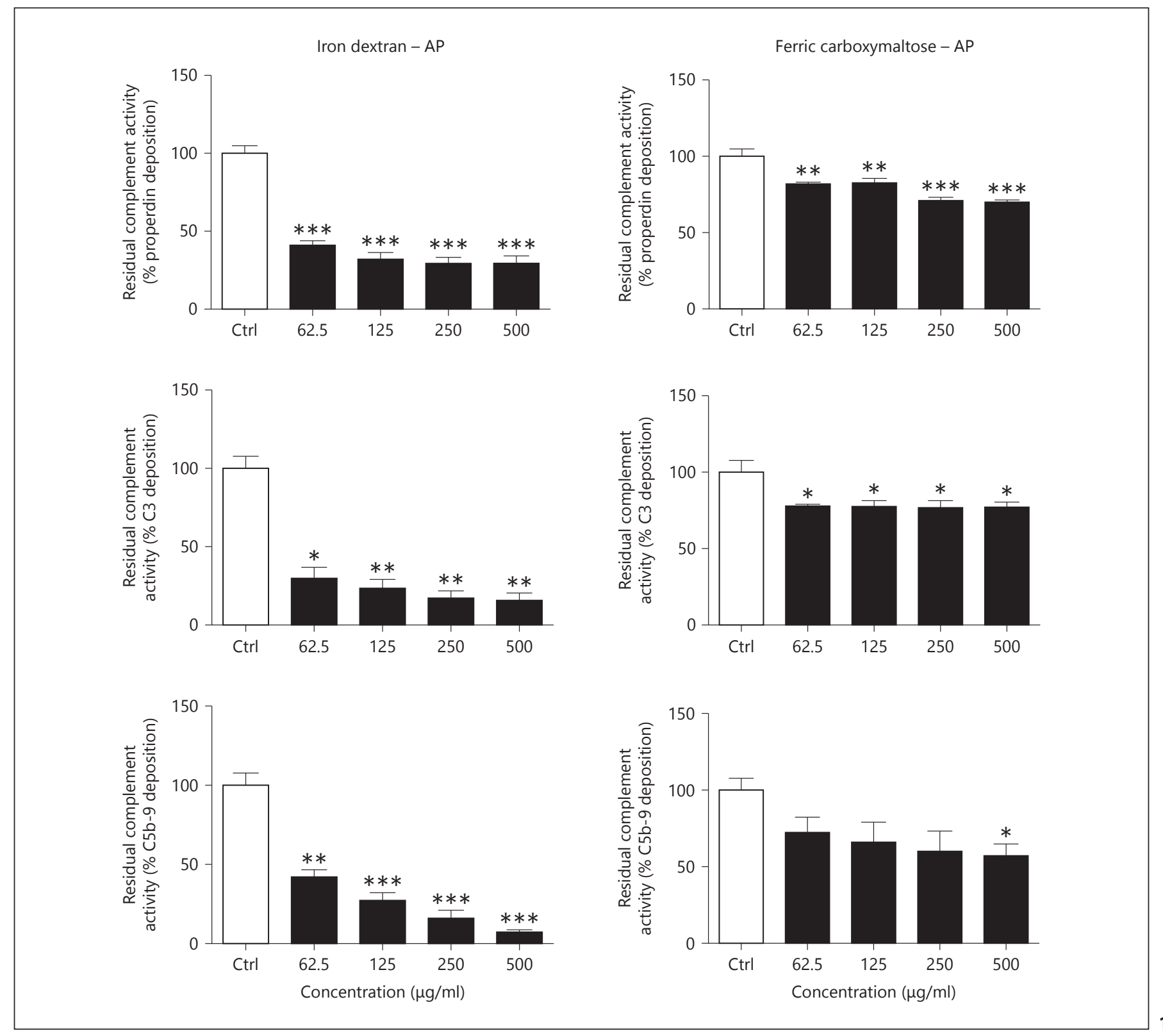

3.1.3 Decreased Residual Activity of the AP by Iron Dextran and Ferric Carboxymaltose

Lastly, residual activity of the AP was analyzed (table 1). The addition of iron dextran and ferric carboxymaltose caused a significant reduction in residual complement activity at the level of C5b-9 generation (fig. 1). In accordance, pre-incubation with iron dextran and ferric carboxymaltose resulted in a significant dose-dependent reduction of residual complement activity at the level of properdin and C3 deposition $(\mathrm{p}<0.01)$. For iron dextran, deposition of properdin, $\mathrm{C} 3$ and $\mathrm{C} 5 \mathrm{~b}-9$ were lowered by 71,85 and $94 \%$ at $0.5 \mathrm{mg} / \mathrm{ml}(\mathrm{p}<0.01)$ and lowered by 34,24 and $30 \%$ at $0.5 \mathrm{mg} / \mathrm{ml}(\mathrm{p}<0.01)$ for ferric carboxymaltose, respectively. Ferumoxytol, iron sucrose and iron isomaltoside did not affect the complement activity of AP.

\subsection{In-vitro Testing of Complement Activation by IV Iron Drugs}

Next, we investigated whether IV iron preparations can directly activate the complement system. In an ELISA-based set-up, we immobilized the IV iron drugs on the plate and added NHS diluted in buffers that allow the specific activation of CP, LP or AP. Under these 


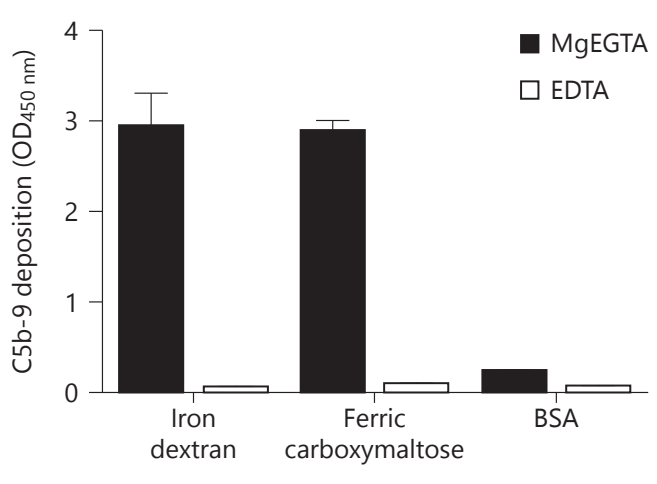

a
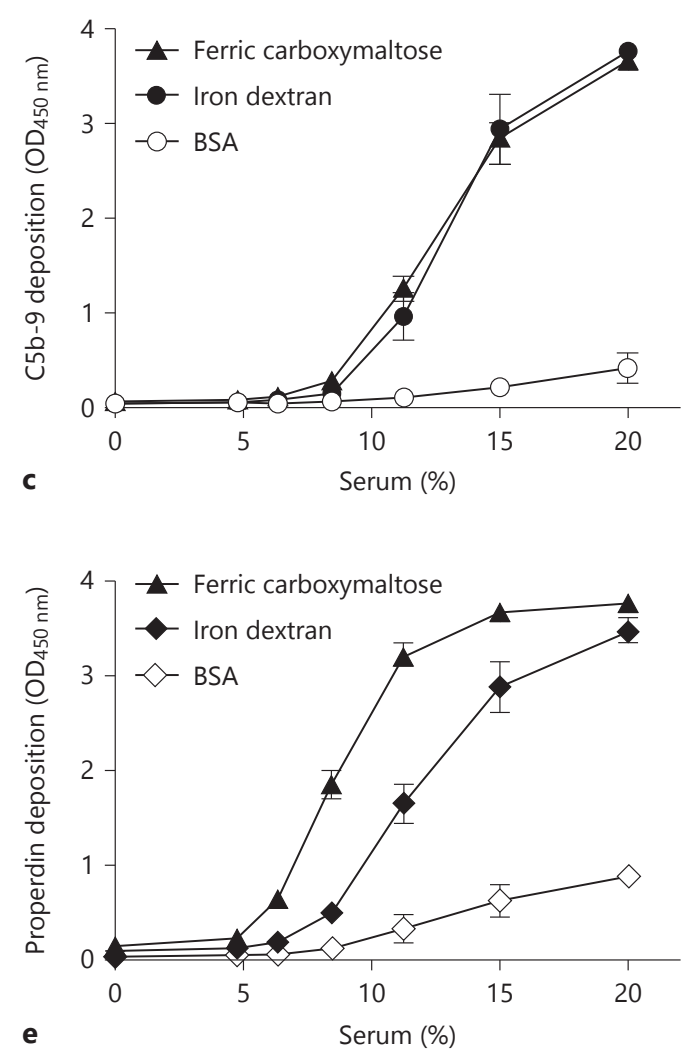
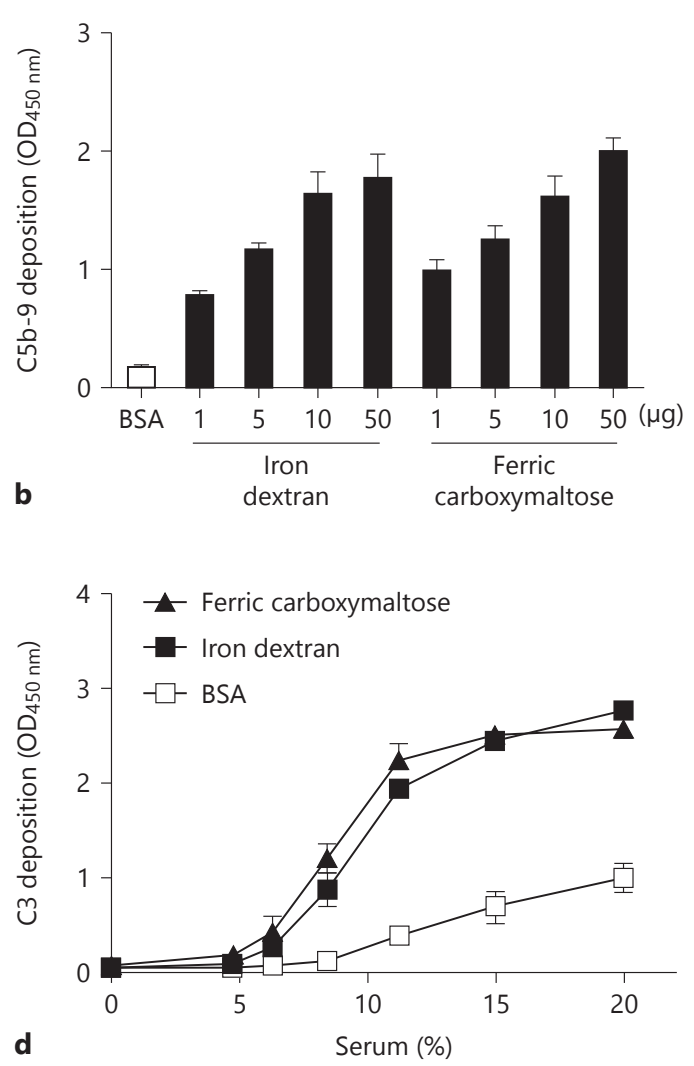

Fig. 2. a-e AP-mediated complement activation on iron dextran and ferric carboxymaltose. a ELISA wells were coated with iron dextran, ferric carboxymaltose at $50 \mu \mathrm{g}$ and $1 \%$ BSA as negative control. Wells were blocked by $1 \% \mathrm{BSA} / \mathrm{PBS}$ for $60 \mathrm{~min}$ at $37^{\circ} \mathrm{C}$. A fixed concentration of $15 \%$ pooled human serum diluted in $\mathrm{GVB}++$ MgEGTA or EDTA was added to the wells with detection by mouse anti-human C5b-9 antibody. Data are shown as mean \pm SEM of 3 experiments. b Iron dextran and ferric carboxymaltose at various concentrations or $1 \%$ BSA were coated to the wells. All coated wells had $1 \% \mathrm{BSA} / \mathrm{PBS}$ added for $60 \mathrm{~min}$ at $37^{\circ} \mathrm{C}$ as a blocking agent. Fifteen percent pooled human serum diluted in GVB++ MgEGTA was added followed by detection using mouse anti-human C5b-9 antibody. c-e Iron dextran and ferric carboxymaltose were coated at $50 \mu \mathrm{g}$ and $1 \% \mathrm{BSA}$ as negative control to the wells. The plate was blocked using $1 \% \mathrm{BSA} / \mathrm{PBS}$ at $37^{\circ} \mathrm{C}$ for $60 \mathrm{~min}$. Increasing concentrations of pooled human serum diluted in GVB++ MgEGTA were added to the wells followed by measuring deposition for C5b-9, C3 or properdin. 
conditions, iron dextran and ferric carboxymaltose had the capacity to activate the AP. Ferumoxytol and iron isomaltoside showed no complement activation for all pathways (table 1). In this set-up, iron sucrose failed to show complement activation for the LP or the CP.

\subsubsection{AP Activation by Iron Dextran and Ferric \\ Carboxymaltose}

We further determined conditions required for iron dextran and ferric carboxymaltose-mediated complement activation. An ELISA plate was coated with iron dextran, ferric carboxymaltose or BSA and then exposed to $15 \%$ pooled human serum diluted in either magnesium ethylene glycol tetraacetic acid (MgEGTA) or EDTA. Subsequently, C5b-9 deposition was assessed. Iron dextran and ferric carboxymaltose coating caused strong C5b-9 depositions compared to BSA controls (fig. 2a). The addition of EDTA completely inhibited complement deposition. Hence, complement deposition was the result of calcium- and magnesium-dependent complement activation. The degree of complement activation was dependent on the concentration of iron dextran and ferric carboxymaltose immobilized on the plate (fig. 2b). Furthermore, we titrated NHS in MgEGTA and showed that C5b-9 depositions were dose-dependent when compared to the negative control, BSA (fig. 2c). Lastly, we tested whether AP activation also involves deposition of other complement components of the AP. We found that similar to C5b-9 deposition, C3 (fig. 2d) and properdin deposition (fig. 2e) occurred in a dose-dependent manner, while no C4 deposition was observed (data not shown). Altogether, these results show that dextran and ferric carboxymaltose-mediated complement activation is only possible under AP conditions.

\subsection{Ex-vivo Analysis of the Effect of IV Iron Drugs on}

Complement Activation in Healthy Volunteers

The effect of IV iron drugs on fluid phase complement activation was determined by incubating IV iron preparation $(0.5 \mathrm{mg} / \mathrm{ml}$ ferrous iron) for $90 \mathrm{~min}$ in human whole blood. Subsequently, complement activation in the samples was determined by measuring soluble C5b-9 (sC5b-9) levels. Increased sC5b-9 levels demonstrate complement activation. Additionally, properdin, MBL and C1q levels were measured to determine which pathway was involved.

\subsubsection{Ex-vivo Terminal Pathway Complement}

Activation by Iron Dextran

The addition of iron dextran to whole blood samples of healthy volunteers led to the activation of vast terminal pathways (fig. 3a). Levels of sC5-b9 were 13-fold higher than in the controls $(p<0.001)$. Incubation with iron sucrose, ferric carboxymaltose, iron isomaltoside or ferumoxytol did not lead to significant complement activation.

\subsubsection{Ex-vivo Complement Activation by Iron}

Dextran Is Mediated via the AP

In order to determine which complement pathway was activated, C1q, MBL and properdin were measured at 0 and 90 min. For iron dextran, a significant decrease in properdin concentration of $42 \%$ was found compared to control $(\mathrm{p}=0.032)$. The concentration of $\mathrm{Clq}$ and $\mathrm{MBL}$ remained largely unchanged (fig. 3b). No significant alterations of in $\mathrm{C} 1 \mathrm{q}, \mathrm{MBL}$ and properdin concentration were found for iron sucrose, ferric carboxymaltose, iron isomaltoside and ferumoxytol.

\subsection{Effect of IV Iron Drugs on Complement in Whole Blood from HD Patients}

Next, we analyzed whether the observed effects of iron dextran can be extrapolated from control subjects without CKD onto HD patients with severe CKD, and whether other iron preparations induce complement activation similar to iron dextran.

\subsubsection{Ex-vivo Terminal Pathway Complement}

Activation by Iron Dextran

Similar to healthy controls, iron dextran led to significant complement activation in whole blood from $\mathrm{HD}$ patients ( $\mathrm{p}<0.001)$, indicated by the marked sC5b-9 generation (fig. 3c). Furthermore, ferric carboxymaltose and iron sucrose led to significant complement activation in HD whole blood but not in healthy controls. However, the complement activation by ferric carboxymaltose and iron sucrose was 2- to 3-fold lower than it was in iron dextran. Iron isomaltoside or ferumoxytol did not lead to significant complement activation.

\subsection{No in-vivo Complement Activation by Current IV}

Iron Treatment in HD Patients

Lastly, we checked if the current IV iron therapy, used at our dialysis unit, leads to in-vivo complement activation in HD patients (fig. 3d, e). Prior to iron therapy, all patients displayed strong complement activation within the first $120 \mathrm{~min}$. The sC5b-9 levels (fig. 3d) increased from $109 \mathrm{ng} / \mathrm{ml}$ (interquartile range (IQR) 85-122) to 247 $\mathrm{ng} / \mathrm{ml}$ (IQR 211-274), while the C3d/C3-ratio (fig. 3e) almost doubled from 7.68 (IQR 5.52-9.92) to 13.04 (IQR 6.55-16.32). Patients then received $100 \mathrm{mg}$ of ferric 

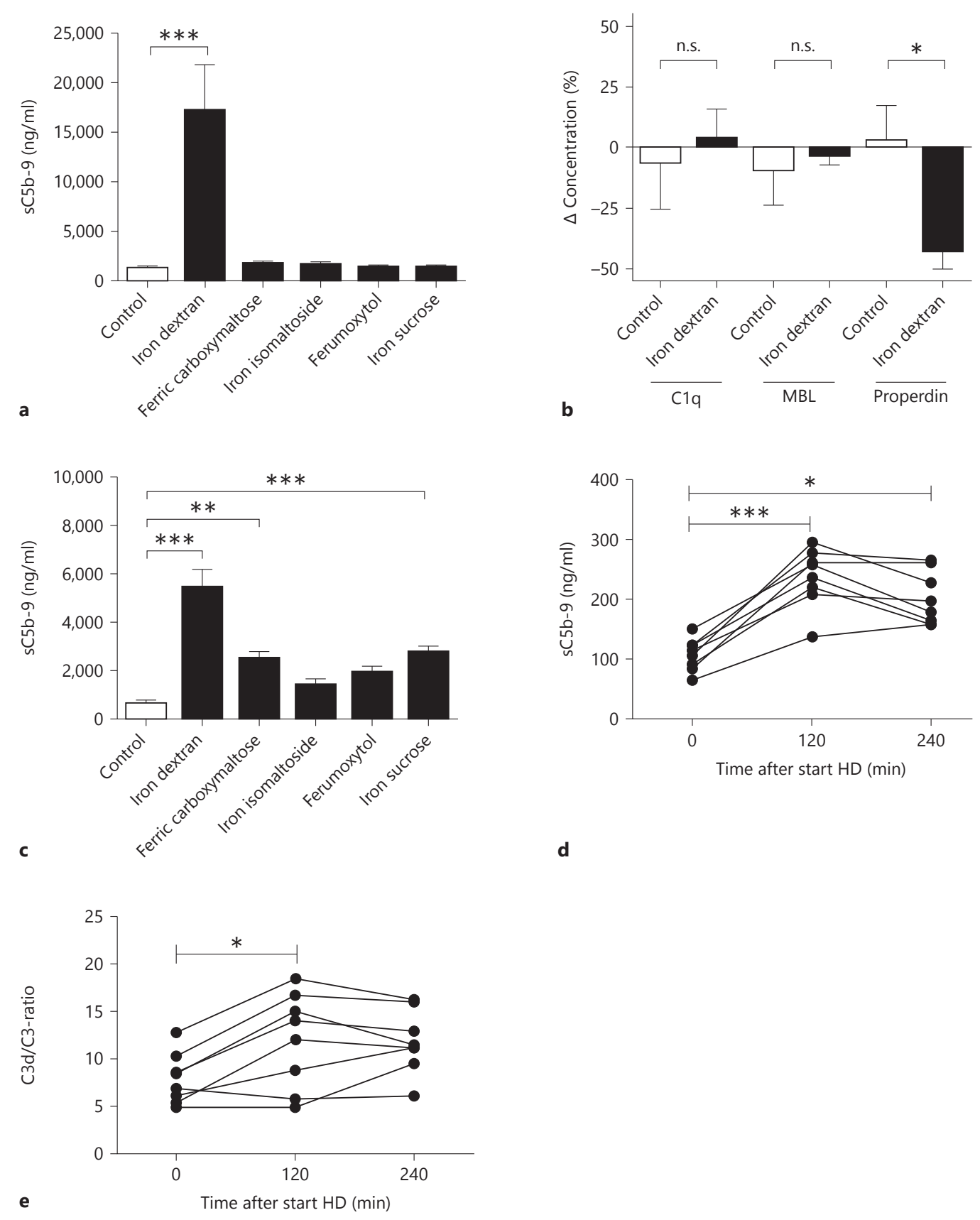

d

Fig. 3. a-e The ex-vivo effect of iron preparations and in-vivo effect of ferric carboxymaltose on complement activation. Whole blood was incubated with $0.5 \mathrm{mg} / \mathrm{ml}$ of iron dextran, Iron sucrose, ferric carboxymaltose, iron isomaltoside and ferumoxytol (x-axis) for 90 min at $37^{\circ} \mathrm{C}$. PBS was used for the controls. a Concentration of $\mathrm{sC5b}-9$ was determined in plasma from healthy controls and used as read-out for complement activation (y-axis). Data are mean and SEM of 5 experiments using different donors each time. b The concentration of C1q, MBL and properdin was determined in samples from healthy controls with $0.5 \mathrm{mg} / \mathrm{ml}$ of iron dextran at 0 and 90 min. The difference in concentration was calculated by dividing the concentration at $90 \mathrm{~min}$, by the concentration at $0 \mathrm{~min}$ and then minus $100 \%$ (y-axis). c Concentration of sC5b-9 was determined in plasma from HD patients (y-axis). Data are mean and SEM of 8 experiments using different donors each time. d sC5-9 levels and e $\mathrm{C} 3 \mathrm{~d} / \mathrm{C} 3$-ratio were determined in HD patients during one dialysis session, in which they received $100 \mathrm{mg}$ of ferric carboxymaltose at $120 \mathrm{~min}$ into the dialysis session. Data are mean and SEM of 8 subjects $\left({ }^{*} \mathrm{p}<0.05,{ }^{* *} \mathrm{p}<0.01,{ }^{* * *} \mathrm{p}<0.001\right)$. 
carboxymaltose intravenously throughout the following $1 \mathrm{~h}$ at $120 \mathrm{~min}$ into the dialysis session. At the end of the dialysis, complement levels remained higher than baseline but did not increase significantly compared to levels at $120 \mathrm{~min}$. Median sC5b-9 levels at $240 \mathrm{~min}$ were $252 \mathrm{ng} / \mathrm{ml}$ (IQR 188-264), while C3d/C3-ratio were 15.22 (IQR 11.40-16.29).

\section{Discussion}

Current EMA-approved IV iron drugs have markedly better safety profiles than the traditional IV iron compounds. However, hypersensitivity reactions still occur and have led to controversy regarding the safety and the risk-benefit ratio of these preparations [2]. Unlike the IgE-mediated reactions by older IV iron compounds, the majority of hypersensitivity reactions by the new IV iron preparations are thought to be caused by CARPA [4-6] The results of our study are the first, to our knowledge, to support this hypothesis by demonstrating the capacity of several IV iron preparations to activate complement in in-vitro and ex-vivo models using blood samples of healthy volunteers and HD patients.

Initially, an in-vitro assay was used to investigate a possible interaction between IV iron and complement in serum. In this set-up, interaction (binding) and complement activation cannot be distinguished. During pre-incubation, the IV iron drug reacts with the complement system. If the IV iron preparation activates complement, this consequently leads to decreased residual complement activity and therefore the deposition on the ELISA plate will be reduced. However, if IV iron binds the complement proteins, then this effect will also reduce complement deposition as the drug is only diluted but not removed after the pre-incubation step.

In order to distinguish between true IV iron-mediated activation and other forms of interaction, ELISA plates were coated with different concentrations of IV iron preparations and fixed concentrations of NHS were added. Complement activation was increased in a dose-dependent manner by iron dextran and ferric carboxymaltose under AP-specific conditions. Combining these results, we can conclude that the reduced complement deposition after incubation with iron dextran and ferric carboxymaltose in NHS in the functional assays was indeed due to complement activation. However, for iron sucrose, we have to consider an alternative explanation such as a direct effect of iron sucrose on $\mathrm{C} 2, \mathrm{C} 4$ or the serine proteases.

Complement Activation by IV Iron
We also tested the capacity of each drug to activate complement in an ex-vivo model. By incubating whole blood with iron, the preparations were not only exposed to serum components but also to blood cells and membrane-bound complement regulatory factors. In line with the previous in-vitro experiments, iron dextran induced significant complement activation, while, surprisingly, ferric carboxymaltose did not. This might be because the functional assays measure complement deposition on a plate and thereby test solid phase activation while the whole blood model tests fluid phase activation by measuring soluble complement activation products. A similar discrepancy has been found for LPS and $\operatorname{IgA}[8,13]$. Furthermore, the whole blood model and the functional assays differ in sensitivity. While coating with the iron preparation and exposing it to NHS serum is a very sensitive test, the whole blood model does not involve dilution of the blood sample and is, therefore, a more physiological approach.

Subsequently, we analyzed the effect of IV iron in a group of HD patients who are regularly receiving IV iron. In the ex-vivo experiments, whole blood from HD patients showed similar activation trends as whole blood from healthy volunteers. In both groups, iron dextran caused a significant increase in $\mathrm{sC} 5 \mathrm{~b}-9$ generation. However, the overall complement activation was lower compared to healthy volunteers. This can be considered a sign of pre-existing chronic complement activation, which is well described in HD patients $[11,12,14]$. Concordantly, in our in-vivo experiments, elevated $\mathrm{C} 3 \mathrm{~d} / \mathrm{C} 3$ ratio and C5b-9 serum levels were measured in blood samples taken from these patients prior to dialysis.

The IV infusion of ferric carboxymaltose did not lead to significant additional complement activation in $\mathrm{HD}$ patients. Both, sC5b-9 levels as well as the $\mathrm{C} 3 \mathrm{~d} / \mathrm{C} 3$ ratio rose during the first half of the dialysis session and then remained consistently elevated from the start of the IV iron administration till the end of the HD session. While these measurements were performed in a small patient group, the results are in line with the ex-vivo findings, which did not indicate strong complement activation capacity for ferric carboxymaltose. Moreover, the slow administration as a continuous infusion over $1 \mathrm{~h}$ reduces the risk of massive complement activation [5, 15]. Lastly, vast complement activation and subsequently relative depletion of complement factors has taken place during the first half of the HD session. We would therefore expect to see more complement activation in non-dialysis CKD patients after IV iron. In addition, we would hypothesize that bolus injection would lead to more complement ac-

Am J Nephrol 2017;45:49-59

DOI: $10.1159 / 000451060$ 
tivation than slow administration. This is supported by previous studies, showing that the rate of infusion is crucial for both the risk of hypersensitivity reactions and complement activation $[5,15,16]$. As none of our patients are currently treated with iron dextran, we were unfortunately not able to test the complement activating properties of this iron preparation in-vivo. To further unravel the effects of different iron preparations in-vivo, a trial comparing the ex-vivo and in-vivo effects of different IV iron drugs in various patient populations would be needed. Nonetheless, since these trials will not be able to observe and compare the very rare clinical severe adverse events, data of observational cohorts including adequate sampling need to be gathered. In addition, further in-vitro studies may help to better understand the mechanism behind hypersensitivity reactions by IV iron preparations.

Clear guidelines exist regarding the maximum dose and minimal duration of administration per IV iron drug. For iron dextran and iron sucrose, the recommended dose is $100-200 \mathrm{mg}$, to be administered intravenously over 2-5 min for 5-10 consecutive HD sessions. Considering an average post-dialysis blood volume of 3,755 \pm $941 \mathrm{ml}$, final blood concentrations would vary between 42 and $71 \mu \mathrm{g} / \mathrm{ml}$. Other IV iron drugs are given in higher doses or administrated more rapidly, resulting in much higher local concentrations at the site of injection than concentrations measured in the peripheral blood $[16,17]$. In addition to that, Geisser and Burckhardt [18] found higher IV iron blood concentrations after repetitive dosing. Thus, concentrations chosen for the experiments are considered physiologically reasonable.

A limitation of our study is the extrapolating of our findings into the clinical setting. Hypersensitivity reactions to IV iron are rare and not in line with the complement activation seen in the in-vitro and ex-vivo results. Thus, an extremely important question that remains to be answered is concerning the difference in frequency of clinically observed adverse events and the frequency and magnitude of complement activation in our in-vitro experiments. Factors such as route and rate of administration and patient characteristic (conditions of pre-existent complement activation) determine the magnitude of complement activation. However, mere activation of the complement system is not sufficient to cause CARPA, but it is a crucial first step in this reaction. In addition, beyond the acute effects, it has been hypothesized that repetitive complement activation, inflammation and oxidative stress may cause endothelial dysfunction and vascular remodeling. Indeed, in an observational study, Michael et al. [19] report an $18 \%$ increase in mortality in HD patients receiving high doses of IV iron. However, due to the observational study design, no conclusion could be drawn regarding the causal relation between IV iron and mortality.

Previous studies defined a 5- to 10-fold increase of complement activation as a realistic predictor for clinical reactions [20]. Given this information, it can be assumed that iron dextran carries a risk of causing CARPA-mediated hypersensitivity reactions. In accordance with our findings, it has been shown that dextran-coated magnetic iron nanoparticles activate the complement system via the AP. These agents are used as an MRI contrast agent and are able to cause severe hypersensitivity reactions in patients. The chemical structure of the iron dextran preparation is similar to this contrast agent [21]. We hypothesize that the iron-carbohydrate nanoparticles have complement-activating properties and not the iron itself, since ferric chloride did not cause significant complement activation (data not shown). In addition, there are several clinical studies stating the higher risk of serious adverse events after the administration of iron dextran formulations $[19,22]$. Recently, Wang et al. investigated the risk of adverse events among the different IV iron drugs. A 3 times higher rate of adverse events was found for iron dextran compared to other IV iron. Also, more anaphylactic reactions were seen after the first administration of IV iron compared to repeated administration [23]. This phenomenon is in line with our results and the description of CARPA [24]. Ferric carboxymaltose also showed complement activating capacity and could shift the regulatory balance in predisposed individuals toward unregulated complement activation.

In conclusion, the present study shows that different IV iron formulations have the in-vitro capacity to activate complement in healthy individuals as well as in HD patients undergoing long-term IV iron treatment. The major finding of this study is that iron dextran significantly activates complement via the AP in-vitro and ex-vivo. In addition, ferric carboxymaltose also activated complement in-vitro via the AP. Furthermore, iron sucrose may interact with complement proteins of the LP and CP, but did not activate complement. Notably, slow infusion of ferric carboxymaltose during HD did not lead to additional complement activation. Our results indicate that current guidelines are efficient at avoiding CARPA by IV iron and explain why these routinely administered drugs show a limited number of adverse events. Our results are the first to our knowl-
58

Am J Nephrol 2017;45:49-59 DOI: $10.1159 / 000451060$
Hempel et al. 
edge, to provide proof of concept of complement activation by IV iron and therefore provide new insights into the pathophysiological mechanism for a well-described adverse reaction to IV iron. Mere activation of the complement system is not sufficient to cause CAR$\mathrm{PA}$, but it is a crucial first step in this reaction. Furthermore, long-term complement activation is known to cause free radical generation and accelerate arteriosclerosis. These findings warrant further translational studies in HD and iron naïve patients in order to gain new insights into the pathophysiological mechanism of these clinical adverse events and to develop a safer treatment.

\section{Acknowledgments}

We thank Anita Meter-Arkema for her excellent technical assistance.

\section{Disclosure Statement}

None of the authors have competing interests to declare.

\section{Financial Support}

This work was financially supported by the Graduate School of Medical Sciences of the University of Groningen.

\section{References}

1 Saran R, Li Y, Robinson B, Ayanian J, Balkrishnan R, Bragg-Gresham J, Chen JT, Cope E, Gipson D, He K, Herman W, Heung M, Hirth RA, Jacobsen SS, Kalantar-Zadeh K, Kovesdy CP, Leichtman AB, Lu Y, Molnar MZ, Morgenstern H, Nallamothu B, O'Hare AM, Pisoni R, Plattner B, Port FK, Rao P, Rhee CM, Schaubel DE, Selewski DT, Shahinian V, Sim JJ, Song P, Streja E, Kurella Tamura M, Tentori F, Eggers PW, Agodoa LY, Abbott KC: US renal data system 2014 annual data report: epidemiology of kidney disease in the United States. Am J Kidney Dis 2015; 65(6 suppl 1):A7.

2 European Medicines Agency: New Recommendations to Manage Risk of Allergic Reactions with Intravenous Iron-Containing Medicines, 2013.

3 Wysowski DK, Swartz L, Borders-Hemphill BV, Goulding MR, Dormitzer C: Use of parenteral iron products and serious anaphylactic-type reactions. Am J Hematol 2010;85: 650-654.

4 Szebeni J, Fishbane S, Hedenus M, Howaldt S, Locatelli F, Patni S, Rampton D, Weiss G, Folkersen J: Hypersensitivity to intravenous iron: classification, terminology, mechanisms and management. Br J Pharmacol 2015;172: 5025-5036.

5 Szebeni J: Complement activation-related pseudoallergy caused by liposomes, micellar carriers of intravenous drugs, and radiocontrast agents. Crit Rev Ther Drug Carrier Syst 2001;18:567-606.

6 Danielson BG: Structure, chemistry, and pharmacokinetics of intravenous iron agents. J Am Soc Nephrol 2004;15(suppl 2):S93-S98.

7 Walport MJ: Complement. First of two parts. N Engl J Med 2001;344:1058-1066.

8 Roos A, Bouwman LH, Munoz J, Zuiverloon T, Faber-Krol MC, Fallaux-van den Houten FC, Klar-Mohamad N, Hack CE, Tilanus MG, Daha MR: Functional characterization of the lectin pathway of complement in human serum. Mol Immunol 2003;39:655-668.

9 Mollnes TE, Brekke OL, Fung M, Fure H, Christiansen D, Bergseth G, Videm V, Lappegård KT, Köhl J, Lambris JD: Essential role of the $\mathrm{C} 5 \mathrm{a}$ receptor in $\mathrm{E}$ coli-induced oxidative burst and phagocytosis revealed by a novel lepirudin-based human whole blood model of inflammation. Blood 2002;100:1869-1877.

10 Trouw LA, Seelen MA, Duijs JM, Benediktsson H, Van Kooten C, Daha MR: Glomerular deposition of $\mathrm{Clq}$ and anti-C1q antibodies in mice following injection of antimouse $\mathrm{Clq}$ antibodies. Clin Exp Immunol 2003;132:3239.

11 Poppelaars F, Gaya da Costa M, Berger SP, Assa S, Meter-Arkema AH, Daha MR, et al: Strong predictive value of mannose-binding lectin levels for cardiovascular risk of hemodialysis patients. J Transl Med 2016;14:236.

12 Poppelaars F, Gaya da Costa M, Berger SP, Assa S, Meter-Arkema AH, Daha MR, et al: Erratum to: Strong predictive value of mannose-binding lectin levels for cardiovascular risk of hemodialysis patients. J Transl Med 2016;14:245

13 Roos A, Bouwman LH, van Gijlswijk-Janssen DJ, Faber-Krol MC, Stahl GL, Daha MR: Human IgA activates the complement system via the mannan-binding lectin pathway. J Immunol 2001;167:2861-2868.

14 DeAngelis RA, Reis ES, Ricklin D, Lambris JD: Targeted complement inhibition as a promising strategy for preventing inflammatory complications in hemodialysis. Immunobiology 2012;217:1097-1105.

15 Auerbach M, Ballard H: Clinical use of intravenous iron: administration, efficacy, and safety. Hematology Am Soc Hematol Educ Program 2010;2010:338-347.

16 Pai AB, Garba AO: Ferumoxytol: a silver lining in the treatment of anemia of chronic kidney disease or another dark cloud? J Blood Med 2012;3:77-85.
17 Chaignon M, Chen WT, Tarazi RC, Bravo EL, Nakamoto S: Effect of hemodialysis on blood volume distribution and cardiac output. Hypertension 1981;3:327-332.

18 Geisser P, Burckhardt S: The pharmacokinetics and pharmacodynamics of iron preparations. Pharmaceutics 2011;3:12-33.

19 Michael B, Coyne DW, Fishbane S, Folkert V, Lynn R, Nissenson AR, Agarwal R, Eschbach JW, Fadem SZ, Trout JR, Strobos J, Warnock DG; Ferrlecit Publication Committee: Sodium ferric gluconate complex in hemodialysis patients: adverse reactions compared to placebo and iron dextran. Kidney Int 2002;61: 1830-1839.

20 Chanan-Khan A, Szebeni J, Savay S, Liebes L, Rafique NM, Alving CR, Muggia FM: Complement activation following first exposure to pegylated liposomal doxorubicin (Doxil): possible role in hypersensitivity reactions. Ann Oncol 2003;14:1430-1437.

21 Banda NK, Mehta G, Chao Y, Wang G, Inturi S, Fossati-Jimack L, et al: Mechanisms of complement activation by dextran-coated superparamagnetic iron oxide (SPIO) nanoworms in mouse versus human serum. Part Fibre Toxicol 2014;11:64.

22 Coyne DW, Adkinson NF, Nissenson AR, Fishbane S, Agarwal R, Eschbach JW, Michael B, Folkert V, Batlle D, Trout JR, Dahl N, Myirski P, Strobos J, Warnock DG; Ferlecit Investigators: Sodium ferric gluconate complex in hemodialysis patients. II. Adverse reactions in iron dextran-sensitive and dextran-tolerant patients. Kidney Int 2003;63:217-224.

23 Wang C, Graham DJ, Kane RC, Xie D, Wernecke $\mathrm{M}$, Levenson $\mathrm{M}$, et al: Comparative risk of anaphylactic reactions associated with intravenous iron products. JAMA 2015;314:20622068.

24 Szebeni J: Complement activation-related pseudoallergy: a stress reaction in blood triggered by nanomedicines and biologicals. Mol Immunol 2014;61:163-173. 